\title{
Researching the Future: The Information Systems Discipline's Futures Infrastructure
}

\author{
Mike Chiasson $^{1}$ and Ola Henfridsson ${ }^{2}$ \\ ${ }^{1}$ Department of Management Science, Management School, \\ Lancaster University, United Kingdom \\ m.chiasson@lancaster.ac.uk \\ ${ }^{2}$ Department of Applied Information Technology, \\ Chalmers University of Technology, Sweden \\ ola.henfridsson@chalmers.se
}

\section{Introduction}

This proceedings for the IFIP 8. 2 working conference on Researching the Future is an attempt to pull together some of our research community's best ideas about how to research the future. The choice of conference theme should be seen as a way to solicit work that can help in building the information system discipline's futures infrastructure. Such an infrastructure is one that provides an initial and developing set of intellectual structures from which IS research can respond to the needs of our future society.

Researching the future is important. As researchers within a discipline focused on the design, adoption, utilization, and effects of information and communication technologies (ICT), we should be well-positioned to contribute to the future shaping of ICT-based practices. If anticipating and influencing the future is something qualitatively different than immediate research relevance, we must then ask whether and how our approaches to inquiry can affect our ability to do so. Such reflection would be valuable in shaping a discipline that is progressive and confident about its role in dealing with questions about the future. Drawing on a healthy debate about research relevance, it is now time to explore, develop, and substantiate how our field can shape and influence the future of ICT-based practices.

The papers covered in this volume are the result of such reflection. Reflecting differences in philosophical foundation, theoretical orientation, and topical emphasis, each deals with the future of ICT in both traditional and novel ways, in illustrating the process and outcomes of ICT applications in contemporary and emerging social settings, as individuals, collectives, firms, and institutions seek to change their future worlds.

\section{Toward a Futures Infrastructure for IS Research}

Some of the ongoing digitally enabled transformations in business, institutions, and societal practices dazzle us with possibilities for improving social welfare, while others suggest that social inequality may be increasing and preserving the status quo. 
Still, there is little doubt that we live in a time when anticipating the future is becoming increasingly difficult. Technologies converge (Lyytinen and Yoo 2002); product life cycles shrink (D'Aveni 1994); institutions evolve (Porter and Teisburg 2006); new learning cultures are born (Thomas and Brown 2011). The multitude of ongoing and parallel changes is vast, which makes the governance of not only enterprises but also some of our society's basic institutions, such as the educational and healthcare systems, increasingly challenging. There are many indications that this complex web of socio-technical changes will continue to evolve as digital technology becomes ever more woven into the fabric of everyday life.

As a result, successful businesses of our time are shifting attention from decisionmaking, planning, and control toward enactment, improvisation, and empowerment. This shift is manifested in attempts to build platform-centric strategies (Ciborra 1996; Tiwana et al. 2010), where the focus is on creating and nurturing the socio-technical capabilities needed for being responsive to emerging opportunities. Such capabilitycreation seems doubly distributed across two dimensions: control and knowledge (Yoo et al. 2010). First, the control over innovation resources is increasingly distributed across actors and stakeholders. Second, the heterogeneity of knowledge sources is increasingly apparent, where digital technology multiplies the space of possible pockets of innovation.

In this period of social transformation, it seems increasingly important for our research discipline to be more than by-standers as our object of study changes the world. To be participants in this change, learning how to research the future is a key ingredient.

Just as the businesses of our time are becoming increasingly infrastructure- centric, it may be time to reflect upon the futures infrastructure of IS research-the practices, logics, and approaches to our research which enable our ability to contribute to the future of IS practice. Important questions arise from this reflection. What can we do in order to be more aware, resilient, and capable of responding and prompting changes in and through our research? How can we adopt infrastructural thinking for making us capable of enacting, improvising, and empowering futures that are responsive to the future needs of our society?

While these are issues partly covered in this book, there are at least three issues that are worth exploring further as we seek to research the future. First, as manifested in the papers included in this volume, we need new conceptual and practical resources for IS research to help us understand what lies ahead. Some argue that our best chance to understand the future lies in understanding the past. Others propose that the adoption of new research methods, especially tailored for future studies, is important. Without a coherent and unambiguous road ahead, a first step toward a futurology of IS is to expand and treat common and unusual positions as resources to engage in the conceptualization, design, and execution of our research.

Second, an IS futures platform would possibly investigate and use the boundary resources of other disciplines. Just as the meanings of a smartphone multiply with infrastructural thinking (not just a phone, but also a navigation system, remote, camera, blood pressure instrument, and so on), it seems likely that the research ecosystem is enriched through the concepts and practices of unrelated disciplines. Neuroscience (Dimoka 2010) and ecological sustainability (e.g., Watson et al. 2010) are recent examples. Moreover, in affecting the future, an IS futures infrastructure also offers its 
boundary resources to emerging knowledge bodies, making it possible for them to tap into our ecosystem (e.g., recent eHealth special issues in IS journals).

Third, an IS futures infrastructure rethinks the prevalent assumption about the individual research hero and the increasing subspecialization prompted by small and isolated collectives. Just as infrastructure owners deal with numerous and emerging networks of stakeholders, a resilient research infrastructure would increasingly be ready to respond to the increasing diversity and changing configuration of the research participants and stakeholders in the future, while still preserving a distance and level-headedness that isn't swept along by fashions and fads. In our view, this suggests the need to think about how to prepare IS researchers for this future, and how the infrastructure for IS research may be able to foster both theoretical stability and practical application as research-participant collectives regroup around important issues emerging in the wake of increasing societal change.

\section{The Review Process}

A total of 36 papers were submitted. The papers were submitted from all three AIS regions, with 7 papers from region 1 (Americas), 24 from Region 2 (Europe, Middle East, and Africa), and 5 from region 3 (Asia and the Pacific). Using a three-tier review structure, all papers went through a rigorous review process where feedback was provided by at least four independent referees including the editorial by one of the program cochairs. While the program chairs managed the 38 papers together, any conflicts-of-interest with a cochair was managed by assigning sole responsibility to the other program chair.

Considering the reviews of members of the program committee, we accepted 16 papers. We also accepted three panels and one workshop presentation on methods for studying the future. The program also includes three plenary sessions with invited speakers.

\section{Overview of the Book}

\subsection{Keynotes}

We would like to show our appreciation of the efforts of our three invited keynote speakers.

Yrjö Neuvo's topic is "Unfogging the Future." He was vice president and chief technology officer for Nokia during the years it was establishing itself as the world's leading mobile phone manufacturer. For many decades, he has been a very influential voice in European research initiatives. Now retired from Nokia, he is based at Aalto University.

The topic of Judith Gregory presentation is "Design for Participation and Negotiation across Logics and Knowledge Communities." She is, and for over 30 years has been, a key figure in the participatory design community. In other words, she engages in making the future together with others. With a very international past, she is currently based at the Illinois Institute of Technology. 
Our third keynote speaker is Youngjin Yoo, with a topic "After the Promised Land: What Do We Do Now?" He is an associate professor in Information Systems and an IBIT Research Fellow at the Fox School of Business and Management at Temple University. His research and teaching interests include digital innovation, experiential computing, design, knowledge management, and virtual teams. You can read his reflections on technology, design, and innovation in everyday experiences in his blog at youngjinyoo.com.

\subsection{Papers}

We wish to thank the authors for the range of approaches taken to the topic of researching the future of information systems. We grouped the articles-somewhat imperfectly-into particular methodological, theoretical, and/or practical approaches to the theme.

We grouped a number of paper focused specifically on how the future and the past are connected and inter-related. Sewchurran and Brown explore the importance of metaphor and analogy across theory and as-lived experience, in transforming the future of IT project management theory and practice, and IS research generally. In a complementary contrast, Aanestad considers how the durability of the past and present is important in understanding and anticipating what the future may hold, and which futures we may wish to hold. Finally, Wynn examines how the future is made "present-at-hand" in corporate projects, and how the future is produced from revisiting the past and realizing its presence within the present. All three papers provide an interesting starting point for thinking about the issues that research about the future may conceptually need to consider.

Another grouping of papers focused on a critical view of the future. Stahl examines discourses emerging from 11 discussions about future ICTs and, by highlighting particular assumptions in the discourse, goes on to critique the implied assumptions in each. In doing so, he provides a basis for examining the unrealized futures beyond these assumptions, which we may wish to pursue instead. Brown examines and explores the people who anticipate and shape the future in organizations-forecasters-and how the supposed foolishness of their activities, as defined within the mathematical forecasting literature, may be seen as an alternative rationality of dealing with the uncertainties of the future in organizations.

Another group of papers examines particular technological futures. Stahl examines the difficulty (if not impossibility) of anticipating specific technological futures, and moves on to consider the need and possibility for participation in shaping the collective future of technology, through approaches such as participatory technology assessment. In contrast to Stahl's epistemology, Andreev, Duane, and O'Reilly examine how perceptions of trust and ease-of-use in m-payment systems affect individuals' willingness to pay and transact using m-payment systems. By examining a future technology using more traditional research approaches, the paper provides an important point of contrast to the open-ended possibilities of Stahl's critique. In a similar vein, Ou, Leung, and Davison examine the growing use of instant messaging tools used in organizations for knowledge management and team performance. Again, as a specific example of researching the future about an important information technology using traditional research methods, the paper provides an important and complementary perspective on 
futures research about particular types of IT, complementing and adding to the debate in this session on the shaping and co-shaping of information systems.

A group of papers examines the future of information technology and work- related practices in health care. Litchner and Venters lead off the health care session using science fiction-a relatively rare approach to researching the future in IS research. They employ science fiction to make a futuristic sense of the evaluation of an electronic prescription system implementation in the national health service (NHS) in the United Kingdom. Pedersen, Ellingsen, and Monteiro consider how the future is standardized through the enactment of both top-down and bottom-up processes in nursing work, and the performativity of standardization beyond the traditional dichotomous view of top-down or futile (bottom-up) views of standardization. Finally, Petrakaki, Cornford, Hibberd, Lichtner, and Barber propose a framework for considering how ICT could affect worker's professional standing in terms of both increasing and decreasing autonomy and capability depending on the effect of ICT on professional roles, work practices, and jurisdictions.

A group of papers examines the future of industrial-institutional practices and outcomes through information technology. Nylén and Holmström consider how latent capabilities in the computing in forestry machinery could transform it into a service sector organization, through the possibilities of open innovation. They make a general claim that open innovation possibilities in an embedded ICT could enable transformation in a number of industrial settings. Crowston examines how insights from the open source community and their use of ICTs may tell us something general about the future of knowledge work-the organization and incentives produced through ICT. Kautz, Bunker, Rab, and Sinnet consider the relations and actions among various companies involved in the production, standardization, and customization of a CRM system, and their use of various open and proprietary positions in producing various niches. They illustrate the range of organizational positions needed to create a rich interorganizational ecosystem around a technological platform, and point to the future of the software industry.

A final group of papers examines the future of critical realism in IS research. Vega and Brown employ critical realism to examine the production and effects of government programs on the diffusion of IT in small and medium sized companies. They illustrate how the social complexity of IS diffusion in the future can be better understood through critical realism (CR). Carlsson explores the past, present, and future use of critical realism in information systems research. Given the position of fallibility in any particular method and study, CR opens the door to many current and future approaches to IS research, and to a never-ending future for IS research practice. A more than fitting conclusion to a special issue on researching the future in IS research.

\subsection{Panels and Workshop}

We have three panels and one workshop in the conference. The first panel by Sawyer, Iivari, Venkatesh, Light and Urquhart examines how our conceptual apparatus for systems design needs to be changed to consider the future of building social software (e.g., online communities) as opposed to role-based software within a single organization. Two questions considered in their panel and discussion are: How do designers 
deal with the tensions of developing more socially complex users of social software versus traditional role-based users, and how do designers grapple with the need to design systems to address social needs versus only functional work activities? Our second panel by Carmel, Avital, Gray, Kallinikos, and King focuses on the importance and relevance of teaching foresight and futuristic thinking in a university curriculum. Their panel focuses on particular controversies in attempting to do so. Our final panel, with Alter, Korpela, Petkov, and Russo, considers the viability of revisiting and reinfusing socio-technical principles and practices in systems development. The panel, through its various questions about the strengths and weaknesses of current approaches, possible extensions, and the current and future trends and issues in design, considers the future form and application of socio-technical approaches in systems development. Our workshop with Gray and Hovav considers three rigorous approaches to futures research in information systems that move beyond simple extrapolation of the present. A fitting conclusion to our volume on the future of IS research about the future.

\section{Conclusion}

In responding to the future of information systems research about the future, the comprehensive and creative response to the call is represented in this volume. However, as a project to produce the infrastructure for futures research in information systems, it is only a beginning within other attempts to tackle the IS researcher's role in shaping the future. Whether it is speculating on particular technological futures, exploring the philosophical foundations for futures research, or critiquing the limited possibilities in visions of the future, the future of IS practice is both potentially limitless and unnecessarily limited. Our only enduring role in the future may be the unstable mixing of observing and understanding what we think is endurable, critiquing what we come to realize is unnecessarily durable, and considering and prompting the IS futures worth having. Our infrastructure for thinking, observing, writing, and participating in dealing with the future may, therefore, be open to continuous exploration and experimentation-a wonderful future indeed!

\section{References}

Ciborra, C.: The Platform Organization: Recombining Strategies, Structures, and Surprises. Organization Science 7(2), 103-118 (1996)

D'Aveni, R.: Hypercompetition: Managing the Dynamics of Strategic Maneuvering. Free Press, New York (1994)

Dimoka, A.: What Does the Brain Tell Us About Trust and Distrust? Evidence from a Functional Neuroimaging Study. MIS Quarterly 34(2), 373-396 (2010)

Lyytinen, K., Yoo, Y.: Research Commentary: The Next Wave of Nomadic Computing. Information Systems Research 13(4), 377-388 (2002)

Porter, M.E., Teisburg, E.O.: Redefining Health Care: Creating Value-Based Competition on Results. Harvard Business School Press, Boston (2006)

Thomas, D., Brown, S.B.: A New Culture of Learning: Cultivating the Imagination for a World of Constant Change (2010) 
Tiwana, A., Konsynski, B., Bush, A.A.: Platform Evolution: Coevolution of Platform Architecture, Governance, and Environmental Dynamics. Information Systems Research 21(4), 685-687 (2010)

Watson, R.T., Boudreau, M.-C., Chen, A.J.: Information Systems and Environmentally Sustainable Development: Energy Informatics and New Directions for the IS Community. MIS Quarterly 34(1), 23-38 (2010)

Yoo, Y., Henfridsson, O., Lyytinen, K.: The New Organizing Logic of Digital Innovation: An Agenda for Information Systems Research. Information Systems Research 21(4), 724-735 (2010) 